\title{
Mitteilungen der Österreichischen IVF-Gesellschaft
}

J. Gynäkol. Endokrinol. AT 2019 · 29:23

https://doi.org/10.1007/s41974-019-0081-0

(c) Springer-Verlag GmbH Austria, ein Teil von Springer Nature 2019

\section{Österreichische IVF GESELLSCHAFT}

Präsident

Prim. Dr. Georg Freude (V.i. S.d.P.)

Sekretariat:

Dr. Roman Haas

Medical Quality GmbH

1190 Wien, Koschatgasse 3

Tel. $+43(0) 14034919$

Fax +43 (0)1 403491915

office@ivf-gesellschaft.at
Sehr geehrte Kolleginnen und Kollegen!

Die Jahrestagung der Österreichischen IVF-Gesellschaft, wieder gemeinsam mit der Österreichischen Gesellschaft für Reproduktionsmedizin und Endokrinologie sowie der Österreichischen Gesellschaft für Sterilität, Fertilität und Endokrinologie, im Oktober 2018 in Innsbruck war gut besucht und reflektierte wiederum das gesamte Spektrum der modernen Fortpflanzungsmedizin.

Die Jahrestagung in diesem Jahr wird vom 17. Oktober bis 19. Oktober in Bregenz stattfinden. Ich ersuche Sie, dieses Datum jetzt schon vorzumerken.

Ab 09. Februar 2019 tritt eine neue EU-Richtlinie in Kraft, die auch die IVF-Institute in Österreich betrifft.

Es geht um folgendes: In ganz Europa müssen ab Februar 2019
Verifizierungssysteme für rezeptpflichtige Humanarzneimittel zum Einsatz kommen. Grundlage dafür sind eine EU-Richtlinie und Delegierte Verordnung betreffend Maßnahmen zur Verhinderung des Eindringens gefälschter Arzneimittel in die legale Lieferkette.

Bevor ein Patient ein Arzneimittel bekommt (unter anderem in einem IVF-Institut), wird dieses aus einem Datensystem ausgelesen. Damit wird sichergestellt, dass es sich um ein reguläres Produkt und nicht um eine Fälschung handelt.

Die Errichtung der entsprechenden Soft- und Hardware obliegt den IVF-Instituten, sodass die in den Instituten an IVF-Patienten abgegebenen Medikamente ab 09. Februar 2019 registriert werden können.

Für die IVF-Institute bedeutet dies wiederum einen zusätzli- chen Kosten- und Verwaltungsaufwand.

Unser Fachgebiet ist publizistisch und ethisch wieder weltweit in den Fokus gerückt durch den Einsatz der Genschere an menschlichen Embryonen durch einen chinesischen Wissenschaftler. In Zukunft sollten die IVF-Gesellschaften vermehrt öffentliche Diskussionen und Aufklärung über diese aktuellen Themen forcieren, damit die Möglichkeiten der Fortpflanzungsmedizin nicht im falschen Licht erscheinen.

In diesem Sinne ist auch die Initiative unseres Vorstandsmitglieds Prof. Kazem Nouri zu erwähnen, der mit Unterstützung der ESHRE im März 2019 die sogenannte „BEI“-Kampagne (Be- wusstsein für Endometriose und Infertilität) starten wird, die auch von der Österreichischen IVFGesellschaft unterstützt wird.

Ein sogenannter „Fertility Bus" wird dann durch Österreich touren, um öffentlichkeitswirksam auf das Thema Endometriose, aber auch Infertilität im Allgemeinen, aufmerksam zu machen.

Am 11.03.2019 wird der „Kickstart“ dieser Aktivitäten im Van-Swieten-Saal in Wien erfolgen. Wir wünschen dieser Kampagne vollen Erfolg.

\section{Mit kollegialen Grüßen}

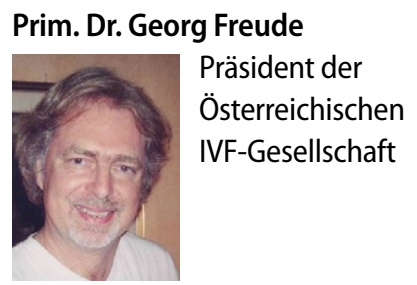

Hier steht eine Anzeige. 